\title{
Evaluation of various procedures transposing global tilted irradiance to horizontal surface irradiance
}

\author{
Caroline Housmans and Cédric Bertrand \\ Royal Meteorological Institute of Belgium, Brussels, Belgium \\ Correspondence to: Caroline Housmans (caroline.housmans@ meteo.be)
}

Received: 6 December 2016 - Revised: 27 January 2017 - Accepted: 31 January 2017 - Published: 9 February 2017

\begin{abstract}
Many transposition models have been proposed in the literature to convert solar irradiance on the horizontal plane to that on a tilted plane. The inverse process, i.e. the conversion from tilted to horizontal is investigated here based upon seven months of in-plane global solar irradiance measurements recorded on the roof of the Royal Meteorological Institute of Belgium's radiation tower in Uccle (Longitude $4.35^{\circ} \mathrm{E}$, Latitude $50.79^{\circ} \mathrm{N}$ ). Up to three pyranometers mounted on inclined planes of different tilts and orientations were involved in the inverse transposition process. Our results indicate that (1) the tilt to horizontal irradiance conversion is improved when measurements from more than one tilted pyranometer are considered (i.e. by using a multipyranometer approach) and (2) the improvement from using an isotropic model to anisotropic models in the inverse transposition problem is not significant.
\end{abstract}

\section{Introduction}

The usual solar radiation parameters measured on ground are the global horizontal irradiance, $G_{\mathrm{h}}$, the direct normal irradiance, $B_{\mathrm{n}}$, the diffuse horizontal irradiance, $D_{\mathrm{h}}$ and the sunshine duration. Traditionally, they are recorded by means of networks of meteorological stations. However, costs for installation and maintenance of such networks are very high and national networks comprise only few stations. The recent deployments of solar photovoltaic (PV) systems offer a potential opportunity of providing additional solar resource information as PV systems with global tilted irradiance, $G_{\mathrm{t}}$, measurements become available in some locations. However, in most PV systems, modules are installed on a fixed plane to reduce installation and operation costs compared to tracking systems. To maximize array output, modules are installed at a tilt close to local latitude, or at some minimum tilt to ensure self-cleaning by rain. $G_{\mathrm{h}}$ estimates at the PV system location requires therefore to convert the tilted solar irradiance recorded to that on a horizontal plane.

If many transposition models have been proposed in the literature (see Yang, 2016 for a review) to convert solar irradiance on the horizontal plane to that on a tilted plane, the inverse process (i.e. converting from tilted to horizontal) is only poorly discussed in literature. The difficulty stems from the fact that the procedure is analytically not invertible. As an example, single-sensor approach involving a numerical search method was proposed by Yang et al. (2013), and evaluated using various combinations of decomposition (i.e. models that separate direct and diffuse solar components from the global one) and transposition models. They found their method sufficiently accurate for small zenith angles, but reported that the conversion error increases exponentially as zenith angle increases. More recently, Marion (2015) presented an iterative method using a modified version of the DIRINT decomposition model (Perez et al., 1992) in combination with the transposition model of Perez et al. (1987) to obtain horizontal irradiance from measurements of a single tilted sensor. Performance of his method was found essentially the same as for the DIRINT model used in the normal calculation mode for $G_{\mathrm{t}}$ measurements recorded at small tilt angle from the horizontal. At larger angles, deviations between modeled and measured $B_{\mathrm{n}}$ and $D_{\mathrm{h}}$ values increase as the tilt angle increases from the horizontal. Lastly, an alternative method based on the Olmo model (Olmo et al., 1999) that presents the property of being analytically invertible was proposed by Killinger et al. (2016). Although the overall performance of the inverted Olmo model was found comparable with the other approaches, the results were slightly worse 
than those obtained by inverting the decomposition and transposition models in combination with an iterative solving process.

The aim of the present study is to (1) evaluate the tilt to horizontal irradiance conversion when using for model input $G_{\mathrm{t}}$ measurements recorded in angular configurations similar to those encountered in Belgian PV systems installations (e.g., with tilt angle as great as 50.79 ${ }^{\circ}$ ) and, (2) determine if, as suggested by Yang et al. (2014), the use of a multipyranometer system could improve the conversion accuracy. The selection of the decomposition and transposition models used in our calculations is based on previous evaluation of popular decomposition and transposition models performance in Uccle by Demain et al. $(2013,2016)$ and Bertrand et al. (2015).

The paper is organized as follows: methods to perform the conversion from tilt to horizontal are presented in Sect. 2. In situ measurements are briefly described in Sect. 3. Performances of the different approaches are evaluated in Sect. 4. Final remarks and conclusions are provided in Sect. 5.

\section{Conversion from tilt to horizontal}

Transposition models have the general form:

$G_{\mathrm{t}}=B_{\mathrm{t}}+D_{\mathrm{t}}+D_{\mathrm{g}}$

where the tilted global solar irradiance, $G_{\mathrm{t}}$, is expressed as the sum of the in-plane direct irradiance, $B_{\mathrm{t}}$, in-plane diffuse irradiance, $D_{\mathrm{t}}$, and the irradiance due to the ground reflection, $D_{\mathrm{g}}$. The direct component, $B_{\mathrm{t}}$, is obtained from:

$B_{\mathrm{t}}=B_{\mathrm{n}} \cos \theta_{\mathrm{i}}=B_{\mathrm{h}} \frac{\cos \theta_{\mathrm{i}}}{\cos \theta_{\mathrm{z}}}=B_{\mathrm{h}} r_{\mathrm{b}}$

with, $B_{\mathrm{n}}$, the direct normal irradiance and, $B_{\mathrm{h}}$, the direct irradiance on a horizontal surface, respectively. $\theta_{\mathrm{i}}$ is the incidence angle and, $\theta_{\mathrm{z}}$, the solar zenith angle, respectively (see Fig. 1 for angles definition). The parameter $r_{\mathrm{b}}=\cos \theta_{\mathrm{i}} / \cos \theta_{\mathrm{z}}$ is a factor that accounts for the direction of the beam radiation. The diffuse component, $D_{\mathrm{t}}$, and the irradiance due to the ground reflection, $D_{\mathrm{g}}$, can be modeled as follows:

$D_{\mathrm{t}}=D_{\mathrm{h}} R_{\mathrm{d}}$

$D_{\mathrm{g}}=\rho G_{\mathrm{h}} R_{\mathrm{r}}$

where, $D_{\mathrm{h}}$, is the diffuse horizontal irradiance, $G_{\mathrm{h}}$, the global horizontal irradiance (i.e. $G_{\mathrm{h}}=D_{\mathrm{h}}+B_{\mathrm{h}}$ ), $R_{\mathrm{d}}$, the diffuse transposition factor, $\rho$, the ground albedo, and, $R_{\mathrm{r}}$, the transposition factor for ground reflection, respectively. In the present study, the isotropic transposition model proposed by Liu and Jordan (1962) (hereafter referred to as LIU model), and the anisotropic models of Hay (1979) (hereafter referred to as HAY model), Skartveit and Olseth (1986) (hereafter referred to as SKA model) and Perez et al. (1987) (hereafter referred to as PER model) have been considered. In addition,

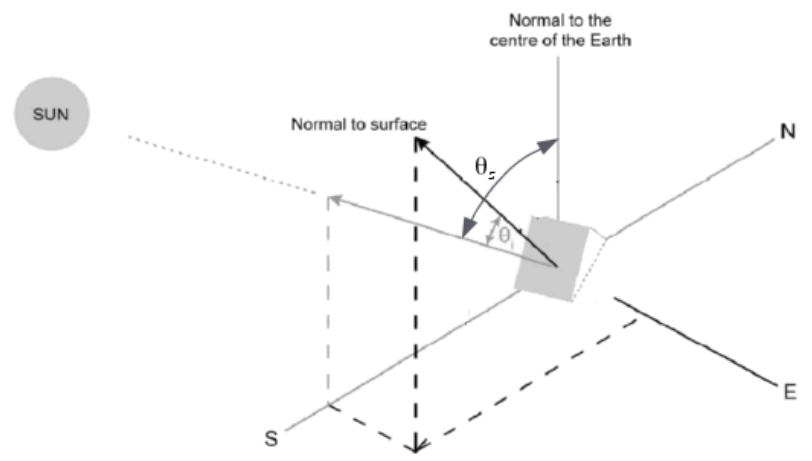

Figure 1. Definition of incidence angle $\theta_{\mathrm{i}}$ on an inclined pyranometer as well as the zenithal solar angle $\theta_{\mathrm{Z}}$.

$R_{\mathrm{r}}$, was modeled under the isotropic assumption (e.g., Gueymard, 2009) and for the sake of simplicity, the foreground's albedo, $\rho$, was fixed to 0.23 (Demain et al., 2013).

\subsection{Single pyranometer approach}

Considering the effective global horizontal transmittance, $K_{\mathrm{t}}$, the direct normal transmittance, $K_{\mathrm{n}}$, and, the diffuse horizontal transmittance, $K_{\mathrm{d}}\left(K_{\mathrm{d}}+K_{\mathrm{n}}=K_{\mathrm{t}}\right)$ :

$$
\begin{aligned}
G_{\mathrm{h}} & =K_{\mathrm{t}} I_{o} \cos \theta_{\mathrm{z}} \\
B_{\mathrm{n}} & =K_{\mathrm{n}} I_{o} \\
D_{\mathrm{h}} & =K_{\mathrm{d}} I_{o} \cos \theta_{\mathrm{z}}
\end{aligned}
$$

where, $I_{o}$, is the extraterrestrial normal incident irradiance, Eq. (1) can be rewritten as:

$G_{\mathrm{t}}=K_{\mathrm{t}} I_{o}\left[\cos \theta_{\mathrm{i}}\left(1-\frac{K_{\mathrm{d}}}{K_{\mathrm{t}}}\right)+\cos \theta_{\mathrm{z}}\left(\frac{K_{\mathrm{d}}}{K_{\mathrm{t}}} R_{\mathrm{d}}+\rho R_{\mathrm{r}}\right)\right]$

Equation (6) indicates that the conversion of $G_{\mathrm{t}}$ to $G_{\mathrm{h}}$ requires the use of a decomposition model to estimate $K_{\mathrm{d}}$ from $K_{\mathrm{t}}$. The decomposition model of Skartveit and Olseth (1987) was used in the present study. This model allows to estimate the direct normal irradiance, $B_{\mathrm{n}}$, from the global horizontal irradiance, $G_{\mathrm{h}}$, through an empirical equation which depends on $K_{\mathrm{t}}$. Equation (6) was solved by a simple iteration procedure. Namely, $K_{\mathrm{t}}$ is first assumed equal to 0.5 and is then adjusted, if needed, using the bisection procedure until the $K_{\mathrm{t}}$ provides calculated $G_{\mathrm{t}}$ value that match the measured $G_{\mathrm{t}}$.

\subsection{Multi-pyranometer approach}

Given $n$ tilted pyranometers (with different inclinations and/or orientations), the inverse transposition problem can be generalized as the matrix form:

$\boldsymbol{x}^{T} \Lambda \boldsymbol{x}+\mathfrak{B}-C=0$

where $\Lambda=\left\{\mathbf{A}_{i}\right\}$ is a $2 \times n \times 2$ third-order tensor, $\mathfrak{B}=\left\{B_{i}\right\}$ is a $n \times 2$ matrix, $C$ is a $n$ column vector, and $\boldsymbol{x}$ a column vector 
with 2 variables:

$$
\begin{gathered}
\mathbf{A}_{i}=\left(\begin{array}{cc}
0 & A_{i} \\
A_{i} & 0
\end{array}\right) \in \mathfrak{R}^{2 \times n \times 2} \\
\mathfrak{B}=\left(\begin{array}{cc}
C_{1} & B_{1} \\
C_{2} & B_{2} \\
\vdots & \vdots \\
C_{n} & B_{\mathrm{n}}
\end{array}\right) \in \mathfrak{R}^{n \times 2} \\
C=\left(\begin{array}{c}
G_{t 1} \\
G_{t 2} \\
\vdots \\
G_{t n}
\end{array}\right) \in \mathfrak{R}^{n} \\
\boldsymbol{x}^{T}=\left(D_{\mathrm{h}} B_{\mathrm{h}}\right) \in \mathfrak{R}^{2}
\end{gathered}
$$

where the coefficients $A_{i}, B_{i}$, and $C_{i}$ depend on the considered transposition model. Note that Eqs. (7) and (8) slightly differ from Yang et al. (2014) due to the change in the column vector $\boldsymbol{x}$ (i.e. $\left(\begin{array}{lll}D_{\mathrm{h}} & B_{\mathrm{h}}\end{array}\right)$ instead of $\left.\left(D_{\mathrm{h}} \lambda\right)\right)$. The least square (hereafter referred to as LS) solution to Eq. (7) is given by:

$\min \left\{P(\boldsymbol{x})=\frac{1}{2}\left\|\boldsymbol{x}^{T} \Lambda \boldsymbol{x}+\mathfrak{B}-C\right\|^{2}: \boldsymbol{x} \in \mathfrak{R}^{2}\right\}$

with $\|$.$\| referring to the Euclidean norm. However, LS is$ hard to solve and a standard technique to resolve Eq. (9) is to use a Newton type iteration method (e.g., Grosan and Abraham, 2008). As an alternative, Eq. (7) can also be solved by minimizing the errors (this approach is hereafter denoted to as EM - Errors Minimization). In this case, the solution is to minimize

$\min \left\{E(\boldsymbol{x})=\sum_{i=1}^{n} \epsilon_{i}^{2}(\boldsymbol{x}): \boldsymbol{x} \in \mathfrak{R}^{2}\right\}$

where, $\epsilon_{i}(\boldsymbol{x})=\left(A_{i} D_{\mathrm{h}} B_{\mathrm{h}}+B_{i} B_{\mathrm{h}}+C_{i} D_{\mathrm{h}}\right)-G_{\mathrm{t} i}$, with $i=$ $1, \ldots, n$ denoting the tilted pyranometer $(n \geq 2)$.

EM approach was selected here to solve Eq. (7) and the Powell's quadratically convergent method (Powell, 1964) was used to perform the minimization. It is a generic minimization method that allows to minimize a quadratic function of several variables without calculating derivatives. The key advantage of not requiring explicit solution of derivative is the very fast execution time of the Powell method. In order to avoid the problem of linear dependence in Powell's algorithm, we adopted the modified Powell's method given in Acton (1970) and implemented in Press et al. (1992).

\section{In situ measurements}

The present analysis is based upon seven months of global solar irradiance measurements performed on the roof of the radiometric tower of the Royal Meteorological Institute of Belgium (RMI) located on the Brussels Uccle plateau (Longitude $4.35^{\circ} \mathrm{E}$, Latitude $50.79^{\circ} \mathrm{N}$ at an elevation of $101 \mathrm{me}-$ ters above sea level). Four data sets of global solar irradiance have been collected from 15 July 2015 to 19 January
2016. The first one was recorded by a Kipp \& Zonen CM11 Secondary Standard pyranometer mounted on the horizontal plane (here after referred to as Plane 0). The second was also recorded by a Kipp \& Zonen CM11 Secondary Standard pyranometer but mounted on a tilted plane of $50.79^{\circ}$ (i.e. corresponding to the RMI radiometric tower's latitude) facing south (here after referred to as Plane 1). For the last two data sets, two additional Kipp \& Zonen CMP-22 Secondary Standard pyranometers were installed on the tower with the same angular configurations as two neighboring residential PV installations, i.e. a tilted plane of $45^{\circ} \mathrm{SW}$ facing and a tilted plane of $50^{\circ}$ facing $E$ (here after referred to as Plane 2 and Plane 3, respectively). Note that all pyranometers are subject to the same calibration and maintenance protocol.

Irradiance measurements were made with a $5 \mathrm{~s}$ time step and then integrated to bring them to a $10 \mathrm{~min}$ time step. All $10 \mathrm{~min}$ data have undergone a series of automated quality control procedures similar to those described in Journée and Bertrand (2011) prior to be visually inspected by a human operator.

\section{Results}

The relative ability of the single and multi-pyranometer approaches to predict horizontal irradiance from the tilted one was estimated by means of two statistical error indexes: Mean Bias Error (MBE) and Root Mean Square Error (RMSE).

$\mathrm{MBE}=\frac{1}{n} \sum_{i=1}^{n}\left(e_{i}\right)$
$\mathrm{RMSE}=\sqrt{\frac{1}{n} \sum_{i=1}^{n}\left(e_{i}^{2}\right)}$

where $e_{i}=\left(G_{i, \mathrm{e}}-G_{i, \mathrm{o}}\right)$ is the residual value; $G_{i, \mathrm{e}}$ are the estimated $10 \mathrm{~min}$ values and $G_{i, \mathrm{o}}$ represent the observed $10 \mathrm{~min}$ measures, e.g. statistical validation being performed on a 10 min basis. A positive MBE (resp. a negative MBE) means than the model tends to overestimate (resp. underestimate) the observed measures. To obtain dimensionless statistical indicators we expressed MBE and RMSE as fractions of mean solar global irradiance during the respective time interval,

$\operatorname{MBE}[\%]=\frac{\mathrm{MBE}}{\bar{M}}$

$\mathrm{RMSE}[\%]=\frac{\mathrm{RMSE}}{\bar{M}}$

where $\bar{M}=\frac{1}{n} \sum_{i=1}^{n}\left(G_{i, \mathrm{o}}\right)$ is the measures mean. It is worth pointing out that for a proper estimation of the error statistics, only radiation data recorded with a solar zenithal angle, $\theta_{\mathrm{z}}$, smaller than $85^{\circ}$ and an incidence angle on the plane 

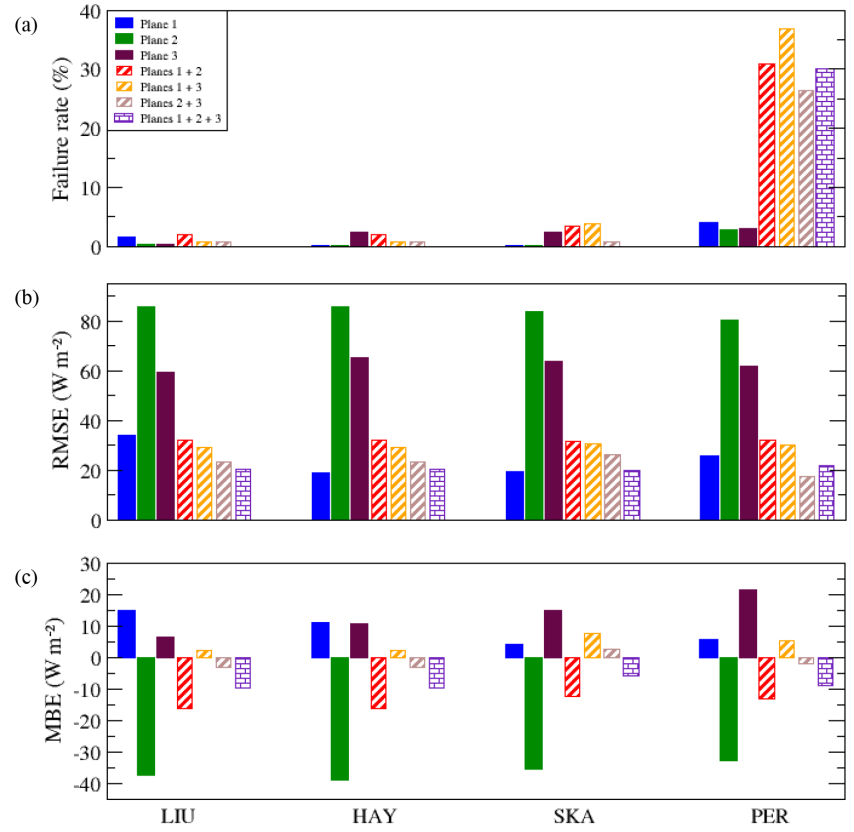

Figure 2. Performance of the four considered transposition models in the tilt to horizontal conversion process. Unsuccessful conversions (a), RMSE (b) and, MBE (c) are reported for a one, two, and three tilted pyranometers system, respectively.

used, $\theta_{\mathrm{i}}$, smaller than $90^{\circ}$ were considered and tilted global solar irradiance records were further restricted to non-zero values. Note that when the retrieved global horizontal solar irradiance was negative or larger than the corresponding extraterrestrial irradiance the inverse transposition problem was considered unsuccessful.

Figure 2 summarizes in terms of failure rate (panel a), RMSE (panel b) and, MBE (panel c) the performance of the inverse transposition for each of the four selected transposition models. Results are reported for a single, two and three tilted pyranometers system, respectively. To evaluate the transposition models on a same basis, only data points conversions that have succeeded for all models are considered in the MBE and RMSE calculations. It is apparent from panel (a) that the PER model exhibits a significant percentage of unsuccessful conversions. Because the PER model coefficients are binned according to the sky clearness parameter, $\epsilon$, the same strategy as in Yang et al. (2014) was adopted here when running this model. Basically, $G_{\mathrm{h}}^{\prime}$ and $D_{\mathrm{h}}^{\prime}$ estimates are calculated for each $\epsilon$ bins using the associated PER model brightness coefficients and then used to determine the corresponding $\epsilon^{\prime}$. If the assumed $\epsilon$ agrees with the calculated $\epsilon^{\prime}$, the $G_{\mathrm{h}}^{\prime}$ and $D_{\mathrm{h}}^{\prime}$ are selected as true estimated $G_{\mathrm{h}}$ and $D_{\mathrm{h}}$ values. Unfortunately, it may appear that none of the retrieved $\epsilon^{\prime}$ values agree with its expected bin, or that more than one retrieved $\epsilon^{\prime}$ values agree with the expected bins, increasing therefore the number of unsuccessful conversions associated to the PER model. This is particularly well apparent in Fig. 2 when more than one tilted pyranometer is involved in the inverse transposition problem. On the other hand, the failure rate reported for the three other models reduces to nothing when three different $G_{\mathrm{t}}$ measurements are involved in the calculations.

With only one tilted irradiance involved in the inverse modeling approach, the tilt angle and the surface's orientation have a major impact on the $G_{\mathrm{h}}$ estimation's reliability irrespective of the considered transposition model. None of the transposition models appears to best perform within the three tilted planes angular configurations. The worst performance in terms of RMSE and MBE are for plane 2 measurements conversion (i.e. RMSE ranging from $80.2 \mathrm{~W} \mathrm{~m}^{-2}$ or $28.7 \%$ to $85.8 \mathrm{~W} \mathrm{~m}^{-2}$ or $30.8 \%$ vs. $19.0 \mathrm{~W} \mathrm{~m}^{-2}$ or $6.8 \%$ to $33.9 \mathrm{~W} \mathrm{~m}^{-2}$ or $12.2 \%$ reported for Plane 1 conversions). Furthermore, Plane 2 conversions underestimate $G_{\mathrm{h}}$ (i.e. MBE ranging from $-39.2 \mathrm{~W} \mathrm{~m}^{-2}$ or $-14.1 \%$ to $-32.7 \mathrm{~W} \mathrm{~m}^{-2}$ or $-11.7 \%$ ) while a slight overestimation of $10.9 \mathrm{~W} \mathrm{~m}^{-2}$ or $0.3 \%$ to $14.9 \mathrm{~W} \mathrm{~m}^{-2}$ or $5.3 \%$ is reported for Plane 1 conversions and an overestimation of $6.5 \mathrm{~W} \mathrm{~m}^{-2}$ or $2.3 \%$ to $21.3 \mathrm{~W} \mathrm{~m}^{-2}$ or $7.6 \%$ for Plane 3 conversions, respectively. As a comparison, Yang et al. (2013) reported RMSE values of roughly 13 and $6 \%$ for the conversion of irradiance data collected by sensors mounted on a $18.3^{\circ}$ tilted NE facing plane and $6.1^{\circ} \mathrm{SW}$ face plane, respectively. However, it is worth pointing out that (1) tilt angles considered in Yang et al. (2013) are much smaller than here and (2) Yang et al. (2014) reported that the RMSE resulting from their conversion using a single tilted sensor NE facing increased from $4 \%$ for a tilt angle of $10^{\circ}$ to $23 \%$ for a tilt angle of $40^{\circ}$.

Figure 2 indicates that the overall performance of the inverse transposition is improved when using two different tilted global irradiance measurements as input to the $G_{\mathrm{h}}$ computation and dependencies to tilt angles and surface orientations are reduced. The LIU isotropic model and the HAY anisotropic model behave similarly with a RMSE (MBE) ranging from $23.0 \mathrm{~W} \mathrm{~m}^{-2}$ or $12.2 \%\left(-3.2 \mathrm{~W} \mathrm{~m}^{-2}\right.$ or $-1.7 \%$ ) for the Plane 2 and Plane 3 measurements conversion to $32.0 \mathrm{~W} \mathrm{~m}^{-2}$ or $16.9 \%\left(-16.2 \mathrm{~W} \mathrm{~m}^{-2}\right.$ or $\left.-8.5 \%\right)$ for the Plane 1 and Plane 2 measurements conversion. Only a slight better performance in term of RMSE and MBE is reported for Plane 2 and Plane 3 measurements conversion performed with the PER model (i.e. a RMSE of $17.5 \mathrm{~W} \mathrm{~m}^{-2}$ or $9.2 \%$ and a MBE of $-1.9 \mathrm{~W} \mathrm{~m}^{-2}$ or $-1.0 \%$ ).

Involving three different tilted irradiance measurements in the conversion process always ensures solutions excepted for the PER model for which the conversion of a bit less than one third of the data points (i.e. 30.4\%) were unsuccessful. All models behave quite similarly in term of RMSE (overall RMSE value of $20.0 \mathrm{~W} \mathrm{~m}^{-2}$ or $7.9 \%$ ) and present a negative bias (i.e. MBE ranging from $-9.7 \mathrm{~W} \mathrm{~m}^{-2}$ or $-3.8 \%$ to $-5.9 \mathrm{~W} \mathrm{~m}^{-2}$ or $\left.-2.4 \%\right)$. In this respect it is worth pointing out that the performance reported for the LIU isotropic transposition model does not differ significantly from the anisotropic transposition models considered in our study. 


\section{Conclusions}

When only a single tilted sensor is used, the conversion can be carried out with a decomposition model coupled with a transposition model to solve the inverse transposition problem. In this case, there is an additional error (additional to the inverse transposition problem) in the predicted horizontal irradiance. In addition, with only one tilted irradiance involved in the inverse modeling approach, the conversion's performance is very sensitive to the tilted pyranometer angular configuration. None of the considered transposition models was found to best perform over the 3 pyranometers mounting plane configurations.

When two (or more) tilted irradiance sensors are involved, only a transposition model is required and the inverse transposition problem can be solved more accurately. In practice, there are certain sun positions for which this procedure still fails to produce a valid estimation of the global horizontal irradiance. Consequently more instruments should be used so as to overdetermine the system. Here it has been shown that three tilted pyranometers set at different orientations is sufficient to guarantee solution for three of the four considered transposition models. Indeed, because of the non bijectivity of the anisotropic transposition model of Perez et al. (1987) the conversion of a percentage of data points can be unsuccessful.

Finally, comparing the performance between the isotropic and anisotropic approaches to the inverse transposition problem indicates that the improvement from using the Liu and Jordan (1962) isotropic model to using anisotropic models is not significant (e.g. a RMSE of $20.0 \mathrm{~W} \mathrm{~m}^{-2}$ or $7.9 \%$ and a MBE of $-9.7 \mathrm{~W} \mathrm{~m}^{-2}$ or $-3.8 \%$ are reported for the LIU model when considering a three tilted pyranometers system vs. a RMSE of $19.6 \mathrm{~W} \mathrm{~m}^{-2}$ or $7.7 \%$ and a MBE of $-5.9 \mathrm{~W} \mathrm{~m}^{-2}$ or $-2.4 \%$ for the best performing SKA anisotropic model) or even inexistent regarding the percentage of unsuccessful conversions (e.g. a failure rate of $0 \%$ is reported for the LIU model considering a three tilted pyranometers system vs. a failure rate of $30.4 \%$ for the PER model).

\section{Data availability}

RMI has not an open data policy, but data can be order at: http://www.meteo.be/meteo/view/en/65239-Accueil.html or by sending an email to ui@ meteo.be.

Competing interests. The authors declare that they have no conflict of interest.
Acknowledgements. This study was supported by the Belgian Science Policy Office (BELSPO) through the BRAIN-be research project: "Solar irradiation from the energy production of residential PV systems-SPIDER", contract No. BR/314/PI/SPIDER.

Edited by: S.-E. Gryning

Reviewed by: two anonymous referees

\section{References}

Acton, F. S.: Numerical Methods That Work; 1990, corrected edition, Washington: Mathematical Association of America, 464467, 1970.

Bertrand, C., Vanderveken, G., and Journée, M.: Evaluation of decomposition models of various complexity to estimate the direct solar irradiance over Belgium, Renew. Energ., 74, 618-626, 2015.

Demain, C., Journée, M., and Bertrand, C.: Evaluation of different models to estimate the global solar radiation on inclined surfaces, Renew. Energ., 50, 710-721, 2013.

Demain, C., Journée, M., and Bertrand, C.: Corrigendum to "Evaluation of different models to estimate the global solar radiation on inclined surfaces", [Renewable Energy 50 (2013) 710-721], Renew. Energ., in press, 2016.

Grosan, C. and Abraham, A.: A new approach for solving nonlinear equations systems, IEEE Trans. Syst. Man Cybern. Part A: Syst. Humans, 38, 698-714, 2008.

Gueymard, C. A.: Direct and indirect uncertainties in the prediction of tilted irradiance for solar engineering applications, Sol. Energy, 83, 432-444, 2009.

Hay, J. E.: Study of shortwave radiation on non-horizontal surfaces, Rep. No. 79-12, Atmospheric Environment Service, Downsview, Ontario, 1979.

Journée, M. and Bertrand, C.: Quality control of solar radiation data within the RMIB solar measurements networks, Sol. Energy, 85, 72-76, 2011.

Killinger, S., Braam, F., Müller, B., Wille-Haussmann, B., and McKenna, R.: Projection of power generation between differently-oriented PV systems, Sol. Energy, 136, 153-165, 2016.

Liu, B. Y. H. and Jordan, R. C.: Daily insolation on surfaces tilted towards the equator, Trans ASHRAE, 67, 526-541, 1962.

Marion, B.: A model for deriving the direct normal and diffuse horizontal irradiance from the global tilted irradiance, Sol. Energy, 122, 1037-1046, 2015.

Olmo, F., Vida, J., Foyo, I., Castro-Diez, Y., and Alados-Arboledas, L.: Prediction of global irradiance on inclined surfaces from horizontal global irradiance, Energy, 24, 689-704, 1999.

Perez, R., Seals, R., Ineichen, P., Stewart, R., and Menicucci, D.: A new simplified version of the perez diffuse irradiance model for tilted surfaces, Sol. Energy, 39, 221-231, 1987.

Perez, R., Ineichen, P., Maxwell, E., Seals, R., and Zelenka, A.: Dynamic global-to-direct irradiance conversion models, ASHRAE Transactions-Research Series, 98, 354-369, 1992.

Powell, M. J. D.: An efficient method for finding the minimum of a function of several variables without calculating derivatives, Comput. J., 7, 155-162, 1964.

Press, W. H., Teukolsky, S. A., Vetterling, W. T., and Flannery, B. P.: Numerical recipes in C. The art of Scientific Computing. Second 
Edition (Reprinted with corrections, 1997). Cambridge University Press, Cambrige, UK, 994 pp., 1992.

Skartveit, A. and Olseth, J. A.: Modelling slope irradiance at high latitudes, Sol. Energy, 36, 333-344, 1986.

Skartveit, A. and Olseth, J. A.: A model for the diffuse fraction of hourly global radiation. Sol. Energy, 38, 271-274, 1987.

Yang, D.: Solar radiation on inclined surfaces: corrections and benchmarks, Sol. Energy, 136, 288-302, 2016.
Yang, D., Dong, Z., Nobre, A., Khoo, Y. S., Jirutitjaroen, P., and Walsh, W. M.: Evaluation of transposition and decomposition models for converting global solar irradiance from tilted surface to horizontal in tropical regions, Sol. Energy, 97, 369-387, 2013.

Yang, D., Ye, Z., Nobre, A. M., Du, H., Walsh, W. M., Lim, L. I., and Reindl, T.: Bidirectional irradiance transposition based on the Perez model, Sol. Energy, 110, 768-780, 2014. 